\title{
Habilidades para la vida en el currículo y práctica de Educación Inicial y Parvularia
}

\author{
Sonia Elizabeth Medrano de Manzano ${ }^{1}$ - Josué Pacheco Castillo ${ }^{2}$
}

Recepción: 03-12-2020 / Aceptación: 25-01-2021

\section{Resumen}

Las habilidades para la vida se consideran como destrezas para la adaptación y comportamiento positivo, que permiten lograr, individualmente, la efectividad para las demandas y retos de cada día durante la vida. Estas son: autoconocimiento, empatía, relaciones interpersonales, toma de decisiones, solución de problemas y conflictos; asertividad, pensamiento creativo, pensamiento crítico, manejo de emociones y sentimientos, manejo de tensiones y estrés. Dichas habilidades están distribuidas en tres áreas: sociales, emocionales y cognitivas; además, se pueden encontrar aportes de las mismas dentro de la forma en la que se imparten, instruyen y desarrollan contenidos dentro de las instituciones de Educación Superior, específicamente a estudiantes en proceso de formación docente de los niveles de Educación Inicial y Parvularia.

En este artículo se exponen los resultados de un estudio mixto, cuya fase cuantitativa fue descriptiva correlacional; mientras que la fase cualitativa, fenomenológica. Con este diseño se pudo obtener un análisis comprensivo de las habilidades para la vida y su incorporación en el diseño curricular del Plan de estudios de las carreras de profesorado y licenciatura en Educación Inicial y Parvularia en El Salvador.

Palabras clave: Habilidades para la vida, currículo, plan de estudios, El Salvador, educación superior, docentes, parvularia, educación inicial.

\begin{abstract}
Life skills are considered as skills for adaptation and positive behavior, which allow to achieve, individually, the effectiveness for the demands and challenges of every day during life. These are: self-awareness, empathy, interpersonal relationships, decision making, problem solving and conflict resolution; assertiveness, creative thinking, critical thinking, management of emotions and feelings, stress and tension management. These skills are distributed in three areas: social, emotional and cognitive; furthermore, contributions of these skills can be found in the way in which contents are taught, instructed and developed within Higher Education institutions, specifically to students in the process of teacher training at the Early Childhood and Kindergarten levels.

This article presents the results of a mixed study, whose quantitative phase was descriptive-correlational, while the qualitative phase was phenomenological. With this design, it was possible to obtain a comprehensive analysis of life skills and their incorporation into the curricular design of the curriculum of the Early Childhood and Kindergarten Education teaching and licensure programs in El Salvador.
\end{abstract}

Key words: Life skills, curriculum, Higher Education, teachers, nursery school, early childhood education.

1. Maestra en Diseño Curricular por la Universidad Don Bosco de El Salvador y Maestra en Atención Integral a la Primera Infancia por la Universidad Pedagógica de El Salvador; email: medrano.manzano@gmail.com; ORCID: https://orcid.org/0000-00019305-8807

2. Doctor en Educación por la Universidad Internacional Iberoamericana y la Universidad Ana G. Méndez. 


\section{Introducción}

Las habilidades para la vida forman parte del currículo y práctica de la Educación Inicial y Parvularia en El Salvador, ya que de acuerdo al Ministerio de Educación de El Salvador $\left(\right.$ MINED) ${ }^{3}$ (2012; p.15) este establece que el maestro de los niveles de Inicial y Parvularia debe tener competencias comunicativas, así como el conocimiento para el desarrollo de habilidades cognitivas, afectivas, sociales $y$ emocionales en la niñez; la resolución de conflictos, la convivencia pacífica y la toma de decisiones asertivas en su proceso formativo y en su ámbito profesional. Estas habilidades se manifiestan en el perfil de egreso del Plan de estudios de las carreras mencionadas.

En este sentido, se puede suponer que la inclusión de las temáticas de manera empírica pueden caracterizar la formación; pero para que su inclusión sea fundamental debe tener pautas, contenidos o metodologías que permitan desplegar las destrezas necesarias, para que los estudiantes en su futuro profesional puedan desarrollarlas con sus beneficiarios. Se espera constatar que en la formación del profesorado se hayan considerado que, las habilidades esperadas en el perfil coincidan con la clasificación de la Organización Mundial para la Salud (OMS) ${ }^{4}$ (1999). Las habilidades para la vida $(\mathrm{HpV})^{5}$ se mencionan como competencias alcanzables dentro del perfil de egreso del profesional como herramientas para la toma de decisiones, y la resolución oportuna y creativa de conflictos. En tal razón, lo esperado es que estas se desarrollen sistemáticamente dentro del proceso formativo.

La iniciativa de la incorporación de las $\mathrm{HpV}$ en el diseño curricular de las carreras de profesorado y licenciatura en Educación Inicial y Parvularia nace junto a los avances que la Educación en la Primera Infancia ha tenido en términos legales; tomando en cuenta los programas y lineamientos del nivel de estudios. Así, colocando a El Salvador al nivel de otros países de Latinoamérica que están prestando atención a niños y adolescentes, cuando se encuentran adquiriendo las habilidades para adaptarse a los medios sociales donde les toca vivir, su integración a los procesos educativos se ve marcada con el Compromiso Hemisférico por la Educación de la Primera Infancia (2007). En este contexto, los ministros de educación establecieron compromisos que garantizan la atención en etapas tempranas de los ciudadanos, garantizando sus derechos, necesidades y deberes.

Lo anterior se encuentra en conjunto con la aplicación de la Ley de Protección Integral de la Niñez y Adolescencia (LEPINA) (2009), cuyo énfasis es realizar

un conjunto de acciones, políticas, planes, programas que con prioridad absoluta se dictan y ejecutan desde el Estado, con la firme participación y solidaridad de la familia y la sociedad para garantizar que to-

3. Los autores también harán referencia a este término mediante su acrónimo.

4. Dentro del documento la autora hará referencia a este término mediante su acrónimo.

5. Con el propósito de acortar este término, la autora utilizará el acrónimo HpV para referirse a él dentro del documento. 
das las niñas y los niños gocen de manera efectiva y sin discriminación los derechos humanos (...) (p.1).

Con esta iniciativa legal, el sistema educativo se transforma institucionalmente para garantizar el nuevo enfoque de protección integral y derecho a la educación de la niñez y adolescencia. Por consiguiente, de acuerdo con el establecimiento actual en la finalidad de la Educación Inicial y Parvularia, es importante incluir las $\mathrm{HpV}$ para garantizar el desempeño en el medio social con recursos propios y el manejo de las emociones para la resolución de problemas.

En este sentido, el currículo para la primera infancia está basado y sustentado en un enfoque de derechos y de desarrollo; superando la visión de etapa preparatoria del ingreso a la escuela básica; y ampliando su propósito al incremento de las posibilidades de desarrollo de todas las potencialidades de los niños. Aquí es que cobra sentido el desarrollo integral del infante; pero estructurar un proceso educativo desde esta perspectiva, "compromete a seguir un marco de intervención en el que se contemple la diversidad y se valore la incidencia de las particularidades biológicas y del contexto sociocultural en el nivel y calidad del desarrollo que cada [niño] alcance" (LEPINA, 2009, p.2).

Las habilidades que se desarrollan a lo largo de la vida se basan en capacidades básicas adquiridas en la primera infancia. El Gobierno de El Salvador, Consejo Nacional de la Niñez y de la Adolescencia (CONNA) y el Comité
Técnico Nacional de Primera Infancia (2018) afirman que,

el desarrollo integral de [los niños] en su primera infancia es una de las prioridades de la agenda económica, social y política de El Salvador; atendiendo a la responsabilidad que tiene el Estado en la implementación de acciones que impacten en el bienestar y la garantía de los derechos de su población. La situación actual de [niños] en su primera infancia impone como desafíos para la familia, el Estado salvadoreño y las instancias corresponsables del cumplimiento de derechos; potenciar el rol protagónico de las familias, cerrar las brechas de acceso y cobertura de servicios y atenciones para la primera infancia, generar ambientes y entornos sanos y seguros; así como los derechos que han sido amenazados o vulnerados (p.13).

Ávila de Parada (2018) enfatiza la importancia de que los gobiernos desarrollen currículos que integren a la familia de los niños en el desarrollo integral de su infancia. El Ministerio de Educación de El Salvador (1999), en el contexto de la Reforma Educativa, "realiza esfuerzos por modernizar su funcionamiento institucional, entregar servicios de calidad y ampliar la cobertura educativa. Dentro de la política 'Mejoramiento de la Calidad Educativa', se desarrollan acciones orientadas a introducir mejoras curriculares, descentralizar servicios educativos, actualizar el marco institucional" (p.5); de manera que la Educación 
Parvularia y Primaria, como afirma Muñoz Moran (2018), pueda mejorarse de manera significativa y cumplir con la integración apropiada de las habilidades para la vida.

Los profesores que deseen implicarse en un programa de prevención deberán adoptar personalmente todos y cada uno de sus principios; concienciándose - en la manera de lo posible - de su repercusión en el futuro de vida de los educandos al impregnarles de entusiasmo. Es necesario determinar qué elementos han de estar presentes, no solo en el currículo para que los programas de los centros educativos puedan aplicarse, sino qué tipo de preparación reciben los futuros docentes de la enseñanza Inicial y Parvularia sobre las $\mathrm{HpV}$. Esto para que estas habilidades se puedan trabajar de manera intencionada, sistemática y explícita; procurando que cada docente sepa aceptarse a sí mismo, y aceptar al otro con sus peculiaridades y particularidades. Solo así, se es capaz de aceptar el mundo que nos toca vivir (Gil Beltrán, 2015).

\section{Análisis del concepto}

El tema de las $\mathrm{HpV}$ ha sido analizado desde varias perspectivas. Se considera que la base de los aprendizajes que se requieren en la escuela deben servir para su aplicación en la vida cotidiana, con función preventiva y de desarrollo (Bizquerra, 2003). El objetivo en el entrenamiento de estas habilidades es aumentar la capacidad de aprendizaje y facilitar una vida más efectiva y satisfactoria. Para ello, se parte de aquellas habilidades psicológicas que facilitan guiar sus propias vidas $y$, al mismo tiempo, poder ayudar a lo además de forma efectiva.

Las HvP, desde un enfoque cognitivo, son comportamientos apropiados y responsables; competencias necesarias para la vida (Remolar, 2002). Estas constituyen un conjunto de capacidades específicas aprendidas en una situación requerida para ejecutar una tarea competente y llegar a comportamientos operativos, repetibles, entrañables y predecibles. El objetivo del programa es que el alumno llegue a incorporar el repertorio de las habilidades necesarias para desenvolverse, y saber solucionar aspectos de conocimiento del trabajo, la familia, el ocio y de su propia vida. Esto supone saber cuándo, dónde y con quién aplicar la habilidad adecuada (Beltrán, 2015). Actualmente, El Salvador requiere como parte de la Educación Inicial y Parvularia la inclusión de HpV. Sin embargo, en la formulación de la programática de contenidos no se evidencia la inclusión de estos temas en la formación de los futuros educadores salvadoreños; por lo que se encuentra una incongruencia inicial entre las competencias que se esperan lograr y el contenido a desarrollar durante el proceso formativo de estos especialistas. El punto de discusión principal es que las asignaturas no refieren concretamente el conocimiento de las $\mathrm{HpV}$ en el currículo de los futuros docentes; mientras que el perfil de egreso sí lo establece.

Según expone Maldonado (2015), la preparación de los maestros de Educación Primaria es esencial en el desarrollo del niño, ya que se 
estimula el proceso de aprendizaje desde sus etapas tempranas de desarrollo. Esto se fundamenta en la creencia de que las habilidades fundamentales adquiridas en la niñez hacen posible un aprendizaje de por vida. Por lo tanto, el objetivo de la preparación del maestro va dirigido al desarrollo del pensamiento creativo, fomento de la innovación y el aprender a aprender del estudiante, según sus necesidades educativas e intereses individuales. El reto de la preparación del maestro es atemperar la Educación Primaria a los cambios que se generan en una sociedad de conocimiento. Estos cambios son tan rápidos que no van acorde con las transformaciones que requieren los currículos en la formación del profesorado de Educación Inicial y Parvularia.

El objetivo general de este estudio fue analizar como las habilidades para la vida $(\mathrm{HpV})$ se incorporan en el diseño curricular de la carrera de profesorado y licenciatura en Educación Inicial y Parvularia en El Salvador para ser aplicadas en la práctica docente. Con este análisis se espera aportar nuevas líneas investigativas y conceptos, relacionados con la preparación de maestros en sus programas académicos sobre las HpV; y así, mover hacia una reflexión del futuro maestro sobre la importancia de incluir - dentro de sus materias - la enseñanza integrada de estas en los currículos de nivel Inicial y Parvularia.

Para ello se plantearon las siguientes hipótesis de la investigación:

H0: En los programas oficiales de formación inicial docente de las carreras de Educación
Inicial y Educación Parvularia de El Salvador (MINED) no está incorporado puntualmente el tema de las habilidades para la vida $(\mathrm{HpV})$.

H1: En los programas oficiales de formación inicial docente de las carreras de Educación Inicial y Educación Parvularia de El Salvador (MINED) está incorporado puntualmente el tema de las habilidades para la vida $(\mathrm{HpV})$.

\section{Metodología}

La metodología de este estudio fue mixta (cuantitativa-cualitativa). Se entiende que este tipo de investigaciones se justifican porque son complementarias, y proveen diferentes tipos de conocimientos y ventajas al investigador como, por ejemplo, información más detallada y nuevos enfoques del estudio (Boeije, 2010; Eriksson y Kovalainen, 2008, Flick, 2009). La fase cuantitativa fue de tipo descriptiva correlacional; mientras que la fase cualitativa fue de tipo fenomenológica.

La población de este estudio estuvo conformada por estudiantes y docentes formadores universitarios que preparan a maestros en los cursos de profesorado y licenciatura en Educación Inicial y Parvularia de distintas universidades de El Salvador. En este caso, fueron profesores y estudiantes de la Universidad Evangélica de El Salvador, Universidad Modular Abierta y Universidad de Oriente. De la población descrita al principio, se seleccionó una muestra total de 73 participantes utilizando la técnica de muestreo no aleatorio, conocido como muestreo intencional. Del total de participantes, 14 fueron docen- 
tes, que también eran estudiantes de la especialidad en Educación Inicial y Palvularia; y 59 fueron estudiantes de las instituciones de Educación Superior antes descritas. De la cifra total de participantes, solo 18 colaboraron en la entrevista semiestructurada de la fase cualitativa del estudio.

La técnica utilizada fue la encuesta, a través de un cuestionario titulado: Habilidades para la vida en el currículo y práctica de Educación Inicial y Parvularia. Dicho instrumento se constituyó de tres partes:

a. Primera parte: se consultaron datos socio demográficos específicos como: rango de edad, rol, nivel de la carrera, especialidad del docente, estado civil, institución de Educación Superior, municipio y departamento.

b. Segunda parte: fueron 26 preguntas cerradas en modalidad de escala de tres, en las que se indicó si la formación a la cual estuvieron expuestos sobre las $\mathrm{HpV}$ ocurrió: siempre, a veces o nunca. Mediante las respuestas obtenidas, se realizó una evaluación metodológica de las $\mathrm{HpV}$ en docentes y estudiantes, con el propósito de obtener una valoración general sobre ellas.

c. Tercera parte: se añadió una sección sobre preguntas para valorar las destrezas que fomenta la OMS para las HpV. Dentro de ella, los participantes debían indicar en una escala de tres si era: relevante, irrelevante o indiferente. Asimismo, se les entrevistó con respecto a la valoración que hacían sobre las distintas destrezas que les fueron enseñadas durante su formación; y la importancia de estas al ser aplicadas en su salón de clases.

\section{Resultados}

El total de los participantes estuvo dividido de la siguiente manera: $30 \%$ perteneció a la Universidad Evangélica de El Salvador; el 34\% a la Universidad Modular Abierta (sede Sonsonate) y el $36 \%$ a la Universidad de Oriente. Llama la atención que, dentro de la muestra total, el 93\% de los participantes fueron mujeres y un $7 \%$ hombres. Podría suponerse que estas cifras se deben a estereotipos culturales que limitan el deseo de los segundos por dedicar su vida al cuidado de infantes. De acuerdo a Pacheco (2013), históricamente la profesión docente ha sido mayoritariamente femenina, y lo sigue siendo en la actualidad.

Tabla 1. Experiencia laboral de los docentes

\begin{tabular}{|c|c|}
\hline Experiencia & Porcentaje \\
\hline De 0 a 10 años & $43 \%$ \\
\hline De 11 a 20 años & $43 \%$ \\
\hline 21 a 30 años & $\mathbf{7 \%}$ \\
\hline De 31 años o más & $\mathbf{7 \%}$ \\
\hline Total & $\mathbf{1 0 0} \%$ \\
\hline
\end{tabular}

$\mathrm{Al}$ analizar los datos de la tabla 1 se evidencia una experiencia promedio de 12 años entre todos los participantes. Es decir, la mayoría de los docentes que aun reciben formación a nivel Inicial y Parvularia como estudiantes, y que imparten las asignaturas en las carreras de licenciatura y profesorado de Educación 
Inicial y Parvularia, poseen poca o mediana experiencia relacionada con el tiempo de servicio. El perfil sociodemográfico presentado por los participantes coincide con estudios relacionados en donde los jóvenes, sin experiencia previa, son los que eligen estudiar docencia; existiendo una tendencia en cuanto a continuar estudios profesionales o a nivel graduado, a pesar de ya estar laborando en este ámbito (Vaillant y Rossel, 2006; Pacheco, 2004).

En la figura 1 se muestran los datos sobre el rol de estudiante que tienen los participantes, en donde el 78\% cursa la carrera de Profesorado, seguido de los que han optado prepararse en la Licenciatura (21\%). Es evidente que la mayoría de los estudiantes de la mues- tra optan por una carrera más corta que agiliza la inserción laboral; quedando un $1 \%$ de la muestra que prefirió no responder.

La mayoría de los encuestados (63\%) se encuentra entre los 20 a 30 años de edad, mientras que un 3\% oscila entre los 18 a 19 años. $\mathrm{Al}$ analizar los datos, la edad promedio de los participantes fue 25 años. Se deduce que la mayoría de los estudiantes encuestados se encuentra en el rango de jóvenes (ver tabla 2).

Al consultarle a los entrevistados sobre si el currículo desarrollado en sus carreras aborda las $\mathrm{HpV}$ como parte de su formación y aplicación en el aula de clases. De acuerdo al 57\% de los docentes y estudiantes abordados, el currículo aborda las $\mathrm{HpV}$ como parte de su

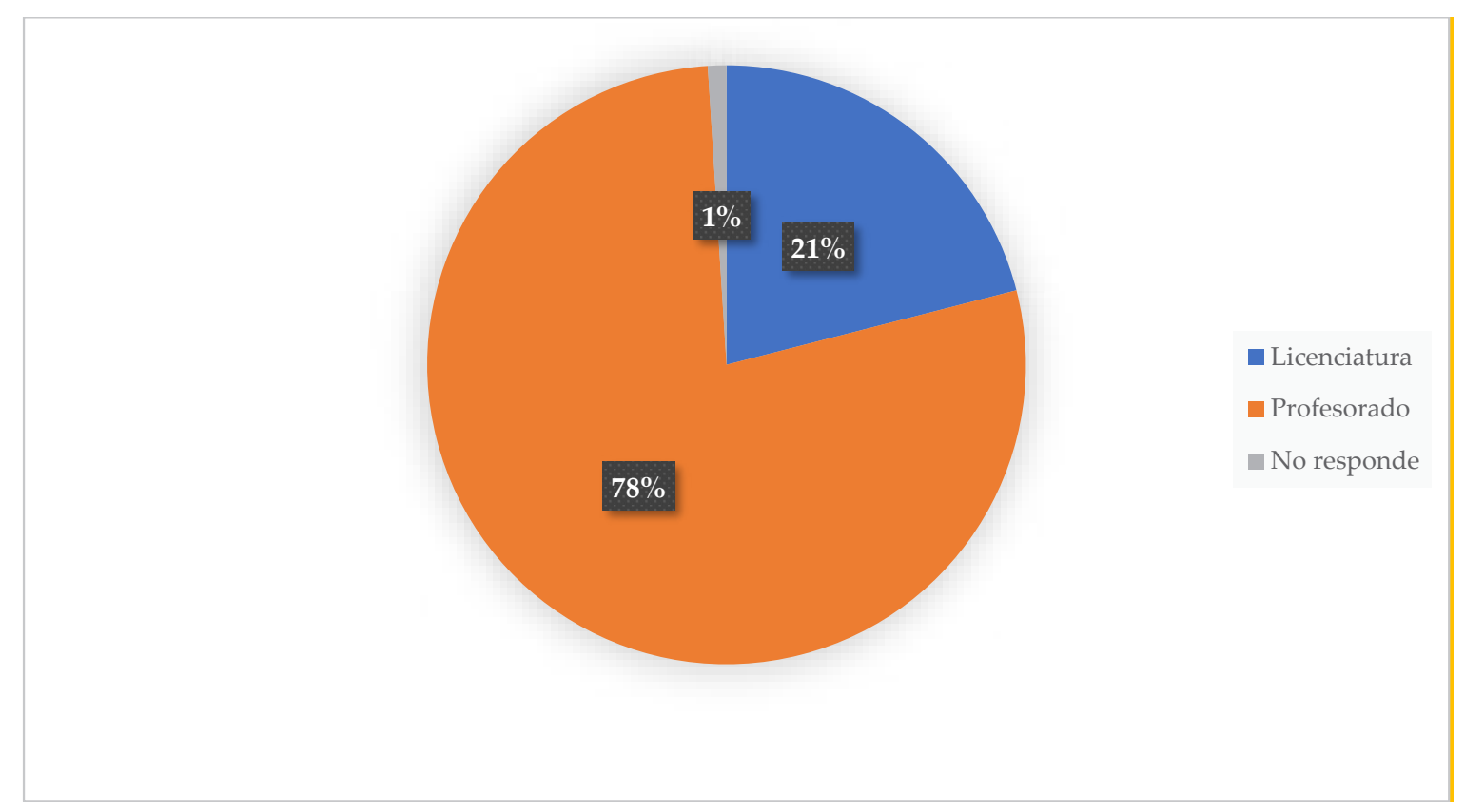

Figura 1. Rol del estudiantes 
Tabla 2. Rango de edad de los estudiantes encuestados

\begin{tabular}{|c|c|}
\hline Rango de edad & Porcentaje \\
\hline De 18 a 19 años & $3 \%$ \\
\hline De 20 a 30 años & $63 \%$ \\
\hline De 31 a 40 años & $19 \%$ \\
\hline De 41 a 50 años & $1 \%$ \\
\hline De 51 a 60 años & $6 \%$ \\
\hline No responde & $8 \%$ \\
\hline Total & $\mathbf{1 0 0} \%$ \\
\hline
\end{tabular}

formación y aplicación en el aula. No obstante, existe un $38 \%$ que indica que este tipo de preparación no es consistente; mientras que un $3 \%$ indica que esto nunca ocurre; dejando a un $1 \%$ que optó por no contestar. Se puede evidenciar que la preparación de los docentes en la carrera de Educación Inicial y Parvularia es inconsistente (ver figura 2).

Como parte del estudio se analizó el perfil del egresado y el currículo utilizado por las universidades para formar a los docentes de la especialidad en Educación Inicial y Parvularia, así como las $\mathrm{HpV}$ recomendadas por la OMS (1999). De acuerdo a lo planteado en el Plan de estudio de las carreras de profesorado y licenciatura, a lo largo de la formación docente, el profesional de Educación Inicial y Parvularia desarrollará características profesionales y personales significativas para la atención educativa a la niñez, en vínculo con la familia y la comunidad. Estas características se han agrupado en tres grandes apartados (dimensiones): conocer, hacer, ser.

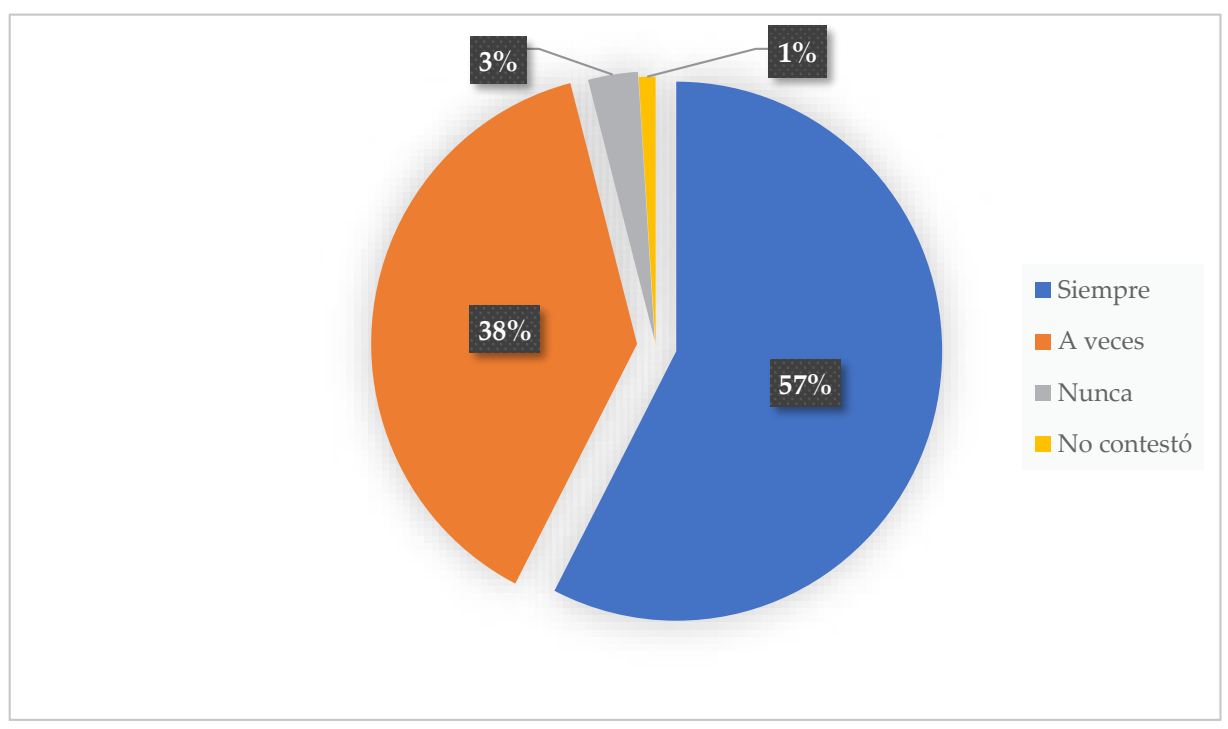

Figura 2. Abordaje de las habilidades para la vida dentro del currículo. 
El Plan de estudios de las carreras de profesorado y licenciatura en Educación Inicial y Parvularia, al ser contrastado con el perfil de egreso de ambas carreras, enuncia un disperso abordaje - dentro de algunas asignaturas - de algunas de las habilidades para la vida como, por ejemplo: la resolución de problemas y conflictos, el manejo de emociones y el pensamiento crítico. Es de reconocer que las diez habilidades para la vida - de acuerdo al planteamiento de la OMS (1993) - no pueden ser abordadas de forma individual, ya que en su mayoría son dependientes entre sí. Asimismo, al aplicar los instrumentos tanto a docentes como estudiantes, una cifra significativa de ellos desconoce sobre el tema; o en algunos casos, su noción es limitada. No obstante, en las tres dimensiones del perfil de egreso del profesional de profesorado y licenciatura las $\mathrm{HpV}$ aparecen como competencias adquiridas. Pero, al revisar minuciosamente, el tema no se encuentra plasmado en la currícula. Los resultados de la figura 3 evidencian que la mayoría de los docentes y estudiantes indican que el hecho que las habilidades para la vida formen parte del currículo y del aula de clases es relevante. A pesar de la importancia de este asunto, un $10 \%$ considera irrelevante que se fomenten y se enseñen $\mathrm{HpV}$ durante su formación de docentes; mientras que un $8 \%$ se muestra indiferente en cuanto a este tema.

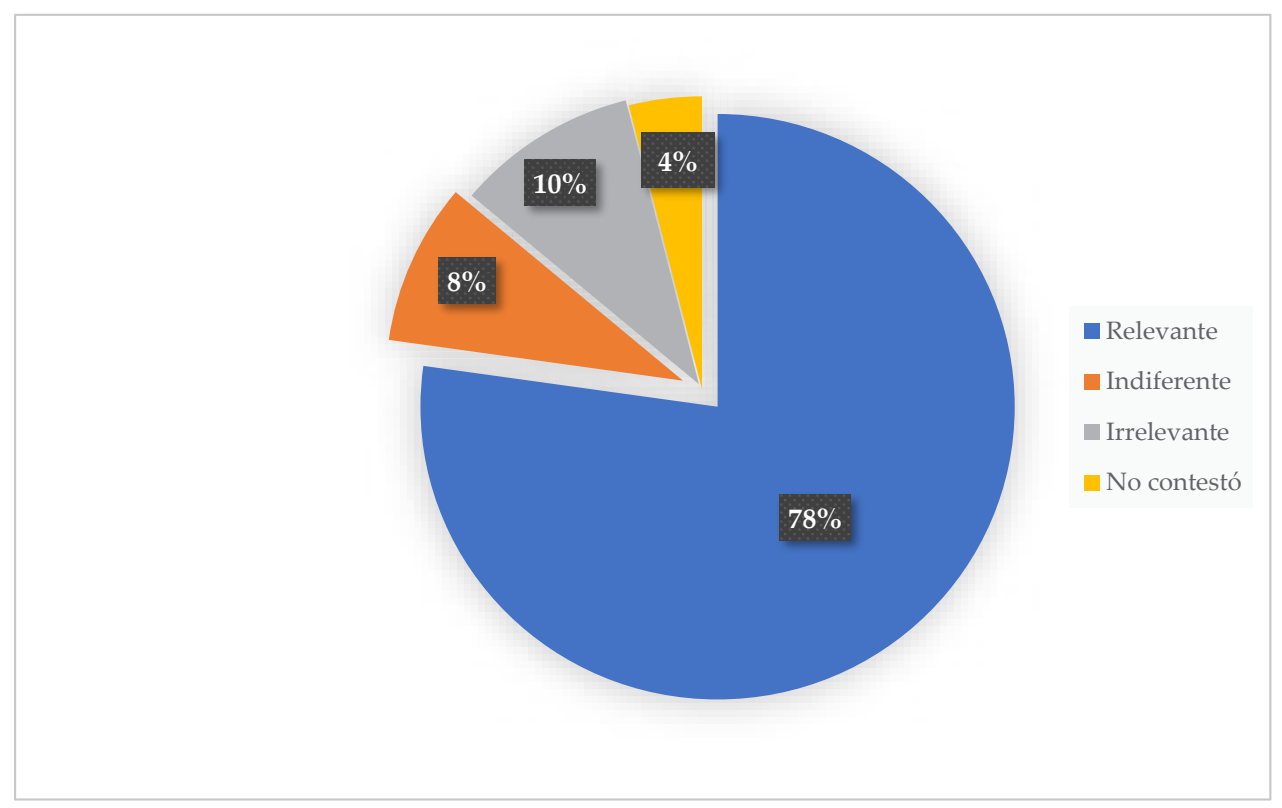

Figura 3. Valoración de las habilidades para la vida como parte de su currículo. 
Para evaluar el conocimiento y práctica de las habilidades para la vida durante el proceso de formación de estudiantes de la carrera de profesorado y licenciatura de Educación Inicial y Parvularia, se tomaron como punto de partida una serie de preguntas:

\begin{tabular}{|c|c|}
\hline Número & Pregunta \\
\hline 1. & $\begin{array}{l}\text { ¿Se fomenta en los estudiantes espacios para la creatividad en diferentes escenarios } \\
\text { de la comunidad educativa? }\end{array}$ \\
\hline 2. & $\begin{array}{c}\text { ¿Considera que debe ser una práctica común, el consultar al inicio de las clases los } \\
\text { gustos o aficiones de los estudiantes? }\end{array}$ \\
\hline 3. & $\begin{array}{c}\text { ¿Se fomenta la habilidad de respuestas asertivas, ante diferentes situaciones que se } \\
\text { le presenten a los estudiantes? }\end{array}$ \\
\hline 4. & $\begin{array}{c}\text { ¿Considera que, durante el proceso de formación, el docente egresado aprende a } \\
\text { promover las habilidades para la vida? }\end{array}$ \\
\hline 5. & $\begin{array}{l}\text { ¿Se utilizan técnicas como la lluvia de ideas para la toma de decisiones, en el espacio } \\
\text { educativo (comunitario-institucional)? }\end{array}$ \\
\hline 6. & $\begin{array}{l}\text { ¿En el proceso de formación, utilizo técnicas para enseñar a los estudiantes la } \\
\text { capacidad de evaluar las consecuencias de sus decisiones y actos? }\end{array}$ \\
\hline 7. & $\begin{array}{l}\text { ¿Observa que sus estudiantes de la especialidad de educación inicial y parvularia, } \\
\text { buscan la innovación en la práctica educativa? }\end{array}$ \\
\hline 8. & $\begin{array}{l}\text { ¿Se realizan, durante el proceso formativo, el desarrollo de actividades que ponen } \\
\text { en práctica el pensamiento crítico? }\end{array}$ \\
\hline 9. & $\begin{array}{c}\text { En el proceso de formación ¿se establece la importancia de las emociones y } \\
\text { sentimientos, en el desarrollo infantil? }\end{array}$ \\
\hline 13. & ¿El trabajo en grupo fomenta las relaciones interpersonales en los estudiantes? \\
\hline 14. & $\begin{array}{l}\text { ¿Se fomenta en el aula, la toma de decisiones, como elemento de apoyo para } \\
\text { alcanzar los logros académicos establecidos? }\end{array}$ \\
\hline 15. & $\begin{array}{l}\text { ¿Se fomenta en los estudiantes actividades metodológicas, para la solución de } \\
\text { situaciones problemáticas con diferentes actores de la comunidad educativa? }\end{array}$ \\
\hline 16. & ¿Convierten los conflictos en el aula en oportunidades de reflexión para todos? \\
\hline 17. & $\begin{array}{c}\text { ¿Se promueve durante el proceso de formación que "Nada es definitivo y que todo } \\
\text { se puede cuestionar?" }\end{array}$ \\
\hline 19. & ¿Considera que las diferencias de opiniones dificultan el trabajo en equipo? \\
\hline 20. & $\begin{array}{l}\text { ¿Se aplica en el aula la habilidad de empatía ante diferentes situaciones de la vida } \\
\text { cotidiana? }\end{array}$ \\
\hline 23. & $\begin{array}{c}\text { ¿Considera necesario aplicar las Habilidades para la Vida en la formación de los } \\
\text { estudiantes de los niveles inicial y parvularia? }\end{array}$ \\
\hline 25. & $\begin{array}{c}\text { ¿Considera oportuno abordar las Habilidades para la Vida en su proceso de } \\
\text { formación como asignatura? }\end{array}$ \\
\hline
\end{tabular}

Figura 4. Preguntas relacionadas al conocimiento y la práctica de las $\mathrm{HpV}$. 
Se consideró que aquellos que respondieron "siempre" dentro de dichas premisas, poseían el conocimiento y la práctica de las $\mathrm{HpV}$; aquellos que respondieron de forma ambivalente (“a veces" y "nunca"), se consideraron que no poseían el conocimiento apropiado sobre las $\mathrm{HpV}$.

Se observa (ver figura 5) que el conocimiento y práctica de las $\mathrm{HpV}$, por parte de los docentes y estudiantes durante su formación, puede catalogarse como deficiente (59\%). Esto es preocupante, ya que son ellos quienes en el futuro se convertirán en especialistas a nivel Inicial y Parvularia; en donde se supone apli- carán dicho conocimiento. Estos resultados coinciden con un estudio realizado por Moreno y De la Herrán (2017) sobre el conocimiento que poseen los educadores en niveles primarios. Estos autores señalan que

el nivel de conocimientos técnicos y pedagógicos de las educadoras de párvulos es extremadamente bajo, lo que se ve agravado por una falsa percepción acerca de su nivel de formación disciplinar y pedagógica. Las conclusiones apuntan a que la profesión de educadora de párvulos y en etapas iniciales tiene un gran desafío formativo por delante (p.245).

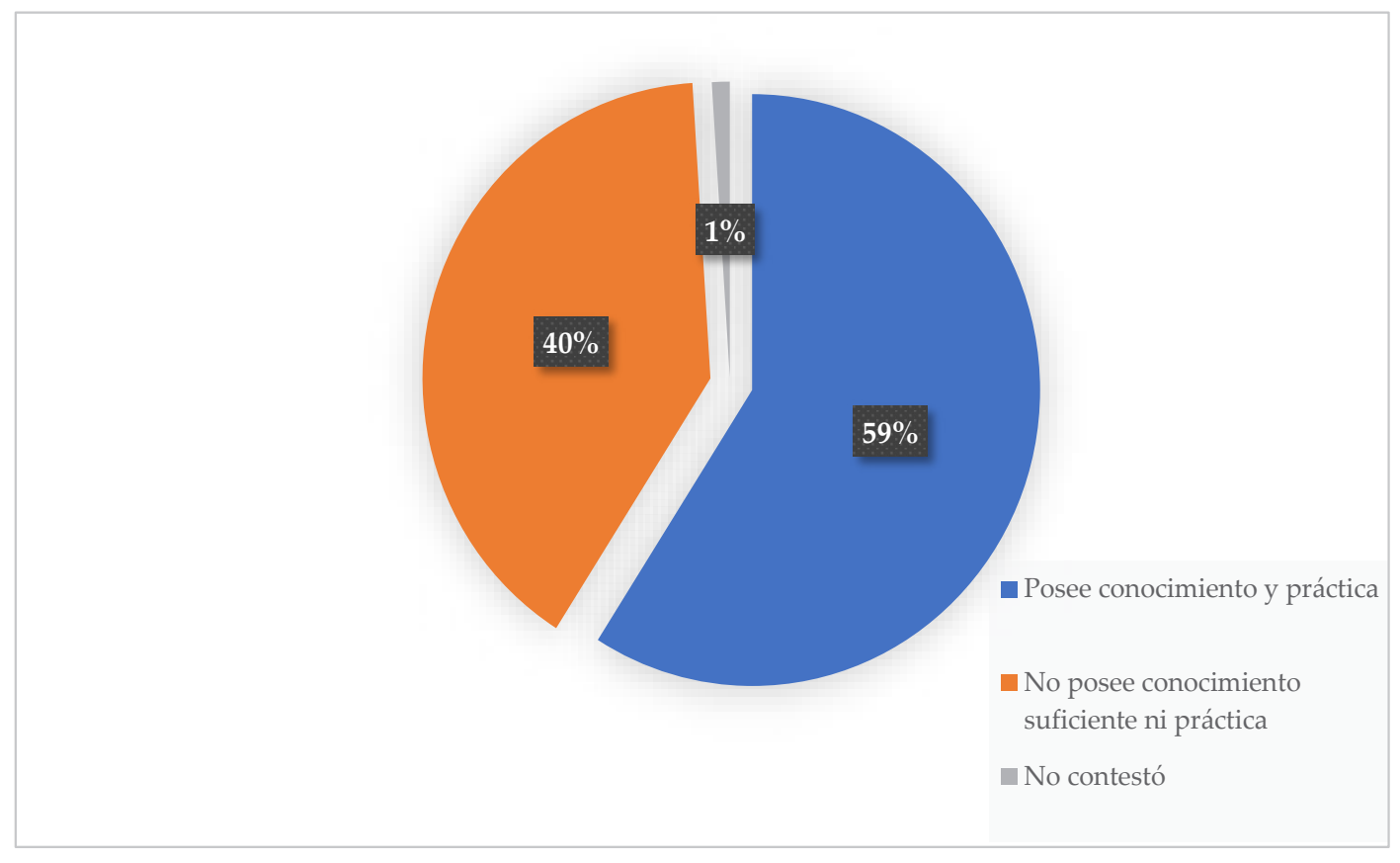

Figura 5. Conocimiento y práctica de las habilidades para la vida durante el proceso de formación de educación inicial y parvularia. 


\section{Análisis de la hipótesis del estudio}

Para medir la hipótesis propuesta para esta investigación se analizaron los hallazgos de la segunda y la tercera parte del cuestionario y comparar los datos de la parte cuantitativa del estudio. Por último, se analizaron cuáles eran las destrezas para la vida que promueve la OMS, y que claramente se identifican dentro del currículo para la especialidad de Educación Inicial y Parvularia (ver figura 6).

Es notable que, en de los programas oficiales de las tres universidades participantes dentro del estudio, no existe una formación consistente sobre las habilidades para la vida. Ante estos resultados se acepta la hipótesis nula y se rechaza la hipótesis de investigación.

\section{Discusión}

Las habilidades para la vida pueden definirse como un conjunto de "habilidades psicosociales para el comportamiento adaptativo y positivo que permiten a las personas lidiar de manera efectiva con las demandas y desafíos de la vida cotidiana" (Maithreyi, 2018, p.5), y facilitan la supervivencia, el afrontamiento, el aprendizaje, el trabajo, jugar, relacionarse con los demás y desarrollarse a sí mismo. Como parte de los marcos curriculares se ha promovido el desarrollo internacional de las $\mathrm{HpV}$, aunque no como se esperaba, ya que han transcurrido más de dos décadas y la preparación de docentes, así como la implantación curricular de las HpV sigue siendo segmentada (Maithreyi, 2015). De ahí que, la capacita-

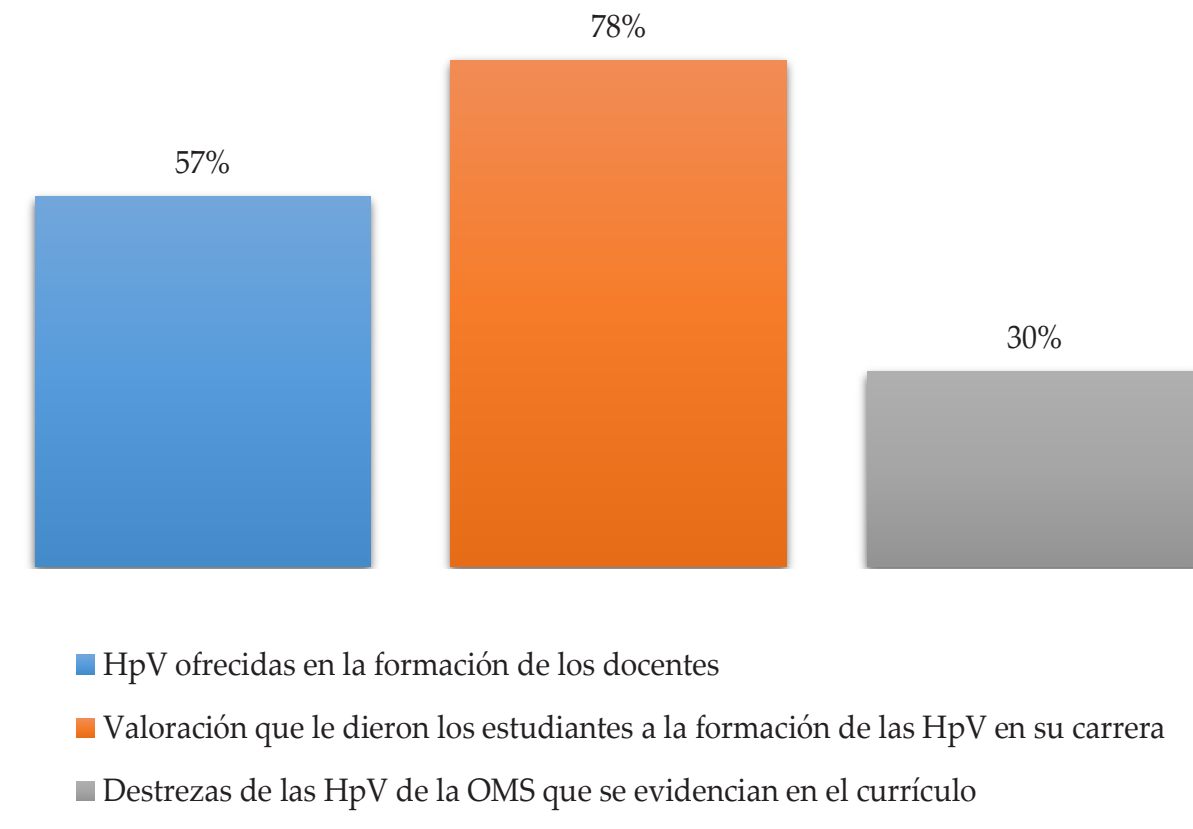

Figura 6. Incorporación de las habilidades para la vida en las carreras de profesorado y licenciatura de Educación Incial y Parvularia. 
ción en habilidades para la vida se ha incluido dentro del Plan de estudios escolar, a través de los esfuerzos de promoción de organizaciones de desarrollo como la Organización Mundial de la Salud (OMS), en varios países del mundo (Maithreyi y Seshadri, 2013).

Cabe enfatizar que, aunque la OMS desde principios de la década de 1990 ha tratado de promover el desarrollo de la niñez, por medio de la enseñanza de las habilidades para la vida en las escuelas, esto todavía no se ha logrado en su totalidad. Se necesita que dichas $\mathrm{HpV}$ sean aplicadas en los currículos de nivel preescolar y escolar, conocidos también como nivel Inicial y Parvularia. Según exponen Abobo y Orodho (2014), aunque varios países han convertido la educación en habilidades para la vida en un componente obligatorio de la Educación Básica, varios factores obstaculizan la enseñanza eficaz de la misma. Una de las situaciones que obstaculizan la implantación correcta de las $\mathrm{HpV}$ en las escuelas es la falta de capacitación a los maestros en este tema; lo que indica un bajo nivel de preparación por parte de estos en ese aspecto. Otros aspectos son la actitud del maestro, la falta de disponibilidad y la adecuación de los recursos de enseñanza y aprendizaje.

Acevedo, Londoño-Vásquez y RestrepoOchoa (2017) señalan la eficacia de exponer a los estudiantes a las $\mathrm{HpV}$, ya que les permiten superar los retos de la vida cotidiana. Los hallazgos sugieren que es pertinente el acompañamiento, por parte de los diferentes actores administrativos y académicos en los primeros semestres, en pro de fortalecer las $\mathrm{HpV}$ requeridas por los estudiantes. Esto con la finalidad de obtener logros académicos manteniendo una salud mental positiva. Por su parte, DíazAlzate y Mejía-Zapata (2017) destacan que no solo es suficiente el conocimiento de las habilidades sociales, sino además su aplicación y la manera en que se apropia de esos contenidos para la toma de decisiones. Durlak, Weisberg, Dymnicki, Taylor y Schellinger (2011) establecen una asociación positiva entre estas intervenciones en favor de las $\mathrm{HpV} \mathrm{y}$ la mejora de las competencias psicosociales, así como la mejora de la actitud, la autoestima y la angustia emocional.

Mojarro (2017) asegura que los entrenamientos son efectivos en los estudiantes, cuando estos son expuestos a las habilidades para la vida como estrategia para la atención primaria de conductas adictivas. De hecho, hubo cambios estadísticamente significativos en el conocimiento antes y después de la implementación del taller de habilidades para la vida: los alumnos que asistieron incrementaron su nivel de conocimiento sobre estas.

Se confirma, a través de los estudios planteados, lo que expone Stewart (2013) y Vallés y Vallés (2016), en cuanto a que no todas las habilidades para la vida surgen de forma natural; muchas de ellas deben introducirse para luego fomentarse y enseñarse constantemente en la escuela, a través de un currículo integrado que permita a los estudiantes preescolares adaptarse al contexto social en que viven. Las habilidades para la vida que los maestros de- 
ben enseñar se dividen en pasos pequeños para que los niños comprendan mejor cuáles son las expectativas y cómo cumplirlas con éxito. Estas $\mathrm{HpV}$ ayudan a los niños pequeños a responsabilizarse de sus propias cosas; pero los niños - a menudo - necesitan orientación y recordatorios de parte de sus maestros y sus padres, en el proceso de dominar este tipo de habilidades para la vida.

Con ello, se debe reconocer que el periodo de los tres a los cinco años es fundamental para el desarrollo de las capacidades sociales, ya que el niño vivencia y registra una serie de situaciones que le permiten organizar su mundo social, comprender normas y prohibiciones como expresar sus propios derechos. Esto permite que el niño pueda percibirse a sí mismo y a los otros de un modo más integrado; adquiriendo una competencia social (Papalía et al., 2010).

\section{Contexto de aprendizaje}

Queda claro que la escuela es un escenario ideal para la puesta en práctica de este tipo de currículo, donde el estudiante, aunque muy pequeño, asume un rol protagónico en su aprendizaje. Según Nasheeda, Abdullah, Krauss y Ahmed (2019), los países desarrollados llevan a cabo programas de educación en habilidades para la vida más sistemáticos, promoviendo un comportamiento positivo en los niños y jóvenes. En cambio, la mayoría de los programas de preparación para la vida de los países en desarrollo carecen de ejecu- ción, evaluación y seguimiento sistemáticos, en donde a menudo se llevan a cabo para producir resultados a corto plazo únicamente. Por ello se deben formular políticas e investigaciones sobre el tema, además, de implementar programas efectivos de habilidades para la vida dirigido a los maestros.

Otieno Okech y Role (2015) afirman que el mundo de hoy se enfrenta a numerosos desafíos, como la indisciplina en las escuelas, los conflictos civiles y las privaciones sociales. Life Skills Education (LSE, por sus siglas en inglés $)^{6}$ es una intervención destinada a equipar a los niños y jóvenes con habilidades para la vida. Para estos investigadores existe una correlación significativa entre la enseñanza de LSE y el desarrollo del carácter. La implementación de LSE tiene como principal desafío la necesidad de recibir apoyo administrativo, mayor adiestramiento a maestros y el involucrar a los padres y a la comunidad a reconocer la importancia de la enseñanza de las $\mathrm{HpV}$ en las escuelas primarias.

La enseñanza de las habilidades para la vida en algunos países se ha delegado al Departamentos de Consejería (Kawira, 2012). Como resultado, los alumnos no están debidamente capacitados para desarrollar las competencias psicosociales. Estudios como el de Chirwa (2009) informan que la implementación de la educación en habilidades para la vida se ha enfrentado a numerosos desafíos: desde la preparación de maestros hasta el reconocer

6. Hacia el final del documento, la autora se referirá a este término mediante su acrónimo en inglés. 
por parte de la comunidad que se incluyan las $\mathrm{HpV}$ en el currículo escolar.

En otro estudio realizado por Abobo y Orodho (2014), se identificó una capacitación docente inadecuada: actitudes negativas de los docentes, recursos de enseñanza y aprendizaje impropios, así como el uso incorrecto de métodos de enseñanza. La formación de profesores en la educación en habilidades para la vida no ha sido parte del plan de estudios de formación de profesores en las universidades e instituciones. En esencia, los maestros que se espera que enseñen educación en habilidades para la vida no están completamente equipados con las habilidades relevantes para manejar la asignatura; lo que los vuelve ineficaces en la entrega del contenido de la misma.

De hecho, la contratación de un número suficiente de profesores calificados ha sido un problema en varios países donde se están implantando los currículos de las $\mathrm{HpV}$ en las escuelas (Siringi, 2012). Este es otro de los retos a superar, ya que los maestros a nivel primario poseen deficiencias en el conocimiento y aplicación de las $\mathrm{HpV}$ en la sala de clases. Meyers (2011, citado por Otieno Okech y Role, 2015) informó que los maestros no estaban preparados para enseñar LSE e implementaron este de manera limitada. Debido a la falta de formación, la mayoría de los profesores abordan la enseñanza de la educación LSE de la misma manera que enseñan otras materias. Esto plantea un desafío importante en la implementación exitosa de cualquier plan de estudios escolar.
Eggan y Kauchak (2015) también descubrieron que el apoyo del profesor hacia los alumnos es importante en el proceso de aprendizaje. Los profesores que defienden estos ideales están comprometidos con su trabajo y cubren el plan de estudios dentro del tiempo asignado.

\section{Conclusiones}

En este estudio se evidenció que la preparación de los docentes en la carrera de Educación Inicial y Parvularia es inconsistente; y no alcanza el porcentaje deseado en términos de exposición durante su formación sobre las HpV. Igualmente, quedó demostrado que el Plan de estudios de las carreras de profesorado y licenciatura en Educación Inicial y Parvularia, al ser contrastado con el perfil de egreso de ambas carreras, enuncia el abordaje en contenidos en el descriptor de algunas asignaturas. Sin embargo, al revisar los temas de todas las asignaturas se enuncia un disperso abordaje de algunas de las habilidades para la vida como, por ejemplo: la resolución de problemas y conflictos, el manejo de emociones y el pensamiento crítico.

También se pudo comprobar que, la mayoría de los docentes y estudiantes evalúan como positivo la incursión del tema habilidades para la vida dentro del currículo, y en las clases de las especialidades de Inicial y Parvularia; sin embargo, este tema no se visibiliza, ni como asignatura, taller o seminario dentro de los programas. Los estudiantes en formación en Educación Inicial y Parvularia afirman la necesidad de aprender sobre el tema, dado 
que sus docentes no lo abordan; o al menos no en la forma en cómo debe ser para internalizarlas desde su proceso formativo.

Otro aspecto importante es el bajo nivel de conocimientos técnicos y pedagógicos de las educadoras de párvulos bajo; lo que se ve agravado por una falsa percepción acerca de su nivel de formación disciplinar y pedagógica. La profesión de educadora de párvulos - en etapas iniciales - tiene un gran desafío formativo por delante. Es decir, que existe un acentuado desconocimiento del significado de las habilidades para la vida. Igualmente, se evidencia que en los programas oficiales evaluados de las tres universidades donde se llevó a cabo el estudio, no existe una formación consistente sobre las habilidades para la vida en las carreras de profesorado y licenciatura de Educación Inicial y Parvularia.

Ante estos resultados se acepta la hipótesis nula, la cual expresa que, en los programas oficiales de formación inicial docente de las carreras de Educación Inicial y Educación Parvularia de El Salvador MINED no está incorporado puntualmente el tema de las habilidades para la vida $(\mathrm{HpV})$. Es decir, que existe una inconsistencia en la formación de los docentes en la especialidad de Educación Inicial y Parvularia respecto al contenido y la aplicación de las habilidades para la vida en su diseño curricular. En otras palabras, el Programa de Educación Inicial y Parvularia que se desarrolla en las instituciones de Educación Superior no presenta de forma concreta, en su diseño curricular, un impacto claro en la enseñanza de habilidades para la vida durante el proceso de formación. De hecho, el tiempo clase dentro del desarrollo curricular está marcado por los aspectos cognitivos en un $90 \%$; en contraste con los aspectos formativos dentro de la esfera de las habilidades para la vida.

\section{Referencias}

Abobo, F. y Orodho, J.A. (2014). Life Skills Education in Kenya: An Assessment of the Level of Preparedness of Teachers and School Managers in Implementing Life Skills Education in TransNzoia District, Kenya. IOSR Journal of Humanities and Social Science, 19, 32-44.

Acevedo-Franco, H.; Londoño-Vásquez, D. A. y Restrepo-Ochoa, D. A. (2017). Habilidades para la vida en jóvenes universitarios: una experiencia investigativa en Antioquia. Revista Katharsis, 24, 157-182.

Aguilar-Avilés, G. (1995). Reforma educativa en marcha en El Salvador, Documento I Un vistazo al pasado de la educación en El Salvador, Ministerio de Educación de El Salvador. http:// webdoc.sub.gwdg.de/ebook/diss/2003/fu-berlin/2002/193/kap3.pdf 
Ariel-Mojarro, L. (2017). Entrenamiento en habilidades para la vida como estrategia para la atención primaria de conductas adictivas. Psicología Iberoamericana, 25, 2.

Ávila-de-Parada, A.C. (2018). Estrategia Nacional para el Desarrollo Integral de la Primera Infancia. file://C:/Users/user/AppData/Local/Temp/ENDIPI_2018-2028-1.pdf

Balmore-Pacheco, R. (2013). Políticas docentes en Centro América: Tendencias nacionales: El Salvador. https://prealblog.files.wordpress.com/2013/12/el-salvador-1.pdf

Compromiso Hemisférico por la Educación de La Primera Infancia (2007). Organización de los Estados Americanos (OEA), Cartagena de Indias, Colombia. https://www.mineducacion. gov.co/primerainfancia/1739/article-192478.html/2021/31/01.

Díaz, A.M. y Zapata, S. (2018). La mirada de los adolescentes al modelo de habilidades para la vida. Revista Latinoamericana de Ciencias Sociales, Niñez y Juventud, 16(2), 709-718. doi: https://doi.org/10.11600/1692715x.16205

Durlak, J.A.; Weisberg, R.P.; Dymnicki, A.B.; Taylor, R.D. y Schellinger, K.B. (2011). The impact of enhancing students' social and emotional learning: A meta-analysis of school-based universal intervention. Child Development, 82(11), 405-432. doi:10.1111/j.1467-8624.2010. 01564.x

Eggen, P. y Kauchak, D. (2015). Educational psychology: Windows on classrooms. Upper Saddle River, NJ: Pearson Education.

Gil-Beltrán, J.M.(2015).Enseñanza de las habilidades de vida en la educación primaria. https://ruc. udc.es/dspace/bitstream/handle/2183/6992/RGP_10-12.pdf?sequence=1\&isAllowed=y

Gobierno de El Salvador, Consejo Nacional de la Niñez y de la Adolescencia-CONNA, Comité Técnico Nacional de Primera Infancia. (2018). Estrategia Nacional para el Desarrollo Integral de la Primera Infancia. file://C:/Users/17879/AppData/Local/Temp/ENDIPI_2018-2028.pdf

Kawira, M.L. (2012). School factors influencing the implementation of life skills education in public primary schools in Athi River District, Kenya. Master's Thesis. University of Nairobi, Kenya.

Ley de protección integral de la niñez y adolescencia "LEPINA". (2009). Compendio Unidad Técnica Ejecutiva del Sector Justicia. -- 1a. ed.-- San Salvador, El Salvador: Comisión Coordinadora del Sector Justicia (UTE).

Maestre-Castro, A. B. (2009). Familia y escuela. Los pilares de la educación. Revista digital innovación y experiencias educativas, 14, 1-11 https://archivos.csif.es/archivos/andalucia/ ensenanza/revistas/csicsif/revista/pdf/Numero_14/ANA\%20BELEN_MAESTRE_1.pdf 
Magnuson, K. A.; Meyers, M. K.; Ruhm, C. J. y Waldfogel, J. (2004). Inequality in Preschool Education and School Readiness. American Educational Research Journal, 41(1), 115-157. doi: https://doi.org/10.3102/00028312041001115

Maithreyi, R. (2015). Reconceptualizing life skills education: A critical analysis of ideas around childhood, 'risks', and 'success. PhD Thesis. Manipal University, Manipal, India.

Maithreyi, R. (2018). Childhood as 'risky' and life as 'skills': Social implications of psycho-educational programmes. En: Saraswathi, TS, Menon, S, Madan, A (eds) Childhoods in India: Traditions, Trends and Transformations. London; New York: Routledge, pp. 252-274.

Maithreyi, R. y Seshadri, S.P. (2013). Life skills education: Current challenges and conceptual directions for a programme for resilience. En: Deb, S, Rangaiah, BR, Sia, SK, Dhanalakshmi, D, Ganth, DB, Thangal (eds) Student's Mental Health. Pondicherry, India: Pondicherry University, pp. 184-200

Ministerio de Educación de El Salvador (2012). Plan de estudios de Profesorado y Licenciatura en Educación Inicial y Parvularia. El Salvador: MINED.

Ministerio de Educación (2013). Manual de Asistencia Técnica para la Primera Infancia Vía Familiar Comunitaria (VFC). El Salvador. https://docplayer.es/31073673-Manual-de-asistencia-tecnica-para-la-primera-infancia-via-familiar-comunitaria-vfc.html

Ministerio de Educación de El Salvador (2013). Programas de educación y desarrollo, nivel de Educación Inicial. El Salvador. https://www.mined.gob.sv/jdownloads/Nuevos\%20Programas\%20de\%20Estudio/Educacin\%20Inicial\%20y\%20Parvularia/inicial_final_ii.pdf

Ministerio de Educación de El Salvador (2014). Plan de Estudio de Profesorado y Licenciatura En Educación Inicial y Parvularia. https://paulofreire.oei.es/system/documents/files/000/000/065/original/EDUCACI\%C3\%93N_INICIAL_Y_PARVULARIA. pdf?1519306585

Moreno, F. y De-la-Herrán, A. (2017). Conocimientos Disciplinares y Pedagógicos de las Educadoras de Párvulos en Chile. Profesorado. Revista de Currículum y Formación de Profesorado, 21(1), 233-251. https://www.redalyc.org/articulo.oa?id=56750681011

Muñoz-Morán, C.A. (2018). Prácticas Pedagógicas en el Proceso de Transición hacia la Escuela Inclusiva. Seis Experiencias en El Salvador. Revista Latinoamericana de Educación Inclusiva, 12(1), 95-110. doi: https://doi.org/10.4067/S0718-73782018000100007 
Organización Mundial de la Salud. (1993). Iniciativa Internacional para la Educación en Habilidades para la Vida en las Escuelas. Habilidades para la vida. http://www.habilidadesparalavida.net/modelo.php

Organización Mundial de la Salud. (1999). Guidelines: Life Skills Education Curricula for Schools. Ginebra, Suiza.

Otieno-Okech, D. y Role, E.M. (2015). Implications of life skills education on character development in children: A case of hill school. https://www.researchgate.net/profile/Daniel_Otieno/ publication/281067569_Implications_of_Life_Skills_Education_on_Character_Development_amongst_children_A_case_of_Hill_School/links/578a56ae08ae254b1de0586e.pdf

Vaillant, D. y Rossel C. (2006). Maestros de Escuelas Básicas en América Latina. PREAL. 